\section{Delayed Development of Extrathoracic Lung Herniation after Blunt Thoracic Trauma}

A 51-year-old African American male with history of Chronic Obstructive Pulmonary Disease (COPD) and asthma was transferred to our institution after sustaining blunt thoracic trauma as a result of a truck falling off its jack onto his chest. According to EMS report, the patient was apneic and pulseless after the truck was removed and bystanders initiated CPR on the scene. He was intubated by paramedics, regained his pulses and transferred to a local emergency room where he underwent initial trauma workup and imaging. Initial evaluation revealed a Glasgow Coma Scale of 3T on arrival and no external thoracic abnormalities. Initial chest CT revealed left anterior sixth and seventh rib fractures without pneumothorax, partial collapse of right upper lobe and bilateral lower lobes, $\mathrm{T} 3$ body fracture, and small diaphragmatic hematoma. A repeat chest CT obtained on arrival to our facility showed nondisplaced right fifth and sixth ribs fractures and bilateral pleural effusions without pneumothorax (see Fig. 1). The patient was admitted to the intensive care unit. Within the first 24 hours of hospitalization, his Glasgow Coma Scale increased to 11T; however, attempts at ventilator wean failed, despite several ventilatory maneuvers, including placing the patient on Airway Pressure Release Ventilation on hospital day 4 (see images). He subsequently developed hemoptysis. Bronchoscopy revealed no acute lung abnormality and patient underwent Computerized Tomographic Angiogram (CTA), which ultimately reveled herniation of his right middle lobe through a widened anterior fourth intercostal space (see Fig. 2).

Presented at the Southeastern Surgical Congress 2016 Annual Scientific Meeting, February 5-25, 2016, Atlanta, GA.

Address correspondence and reprint requests to Anthony G. Charles M.D., M.P.H., Associate Professor of Surgery, UNC School of Medicine, Adjunct Associate Professor of Public Health, Gillings School of Global Public Health, University of North Carolina, 4008 Burnett Womack Building, CB 7228, Chapel Hill, NC. E-mail: anthony_charles@med.unc.edu.
On hospital day 6, the patient underwent a right anterolateral thoracotomy, evacuation of large hemothorax, reduction of herniated lung, and primary closure of this widened intercostal space. Although this effectively corrected the patient's anatomical pulmonary abnormality, he also suffered from progressive bilateral pulmonary contusions, which ultimately proved nonsurvivable.

\section{Discussion}

Lung herniation is defined as "a protrusion of any part of one or both lungs through their boundaries, chiefly the thoracic wall, and usually in a sac of parietal pleura under the skin" and was first described by Roland in $1499^{1}$ and further characterized and classified by Morel-Lavallée in the 1800s. ${ }^{1}$ Extrathoracic lung herniation remains an uncommon abnormality, with roughly 300 total cases described in the literature. These anomalies are typically classified into either congenital or acquired lesions. ${ }^{1}$ Congenital lesions, which account for roughly 20 per cent of described cases, result from hypoplasia or agenesis of the ribs or intercostal musculature and generally present in childhood. ${ }^{2}$ Acquired lung hernias result from, in decreasing order of incidence, traumatic, spontaneous, or pathologic processes. ${ }^{3}$ Treatment strategies depend on the underlying etiology as well as anatomic location. Conservative management and observation can be pursued in small, asymptomatic hernias. However, larger, symptomatic hernias or those at risk of incarceration or strangulation require surgical reduction and either primary or mesh repair of the thoracic wall defect. ${ }^{4}$

For lung herniation to develop, there are two essential criteria: a weakness of thoracic wall, which usually occurs anteriorly from the costochondral junction to the sternum, and a concomitant increase in intrathoracic pressure. In a traumatic injury, especially that of the blunt nature, the kinetic energy required to develop a lung herniation must not be underestimated. Therefore, it is important to remember that reduction of the hernia does 


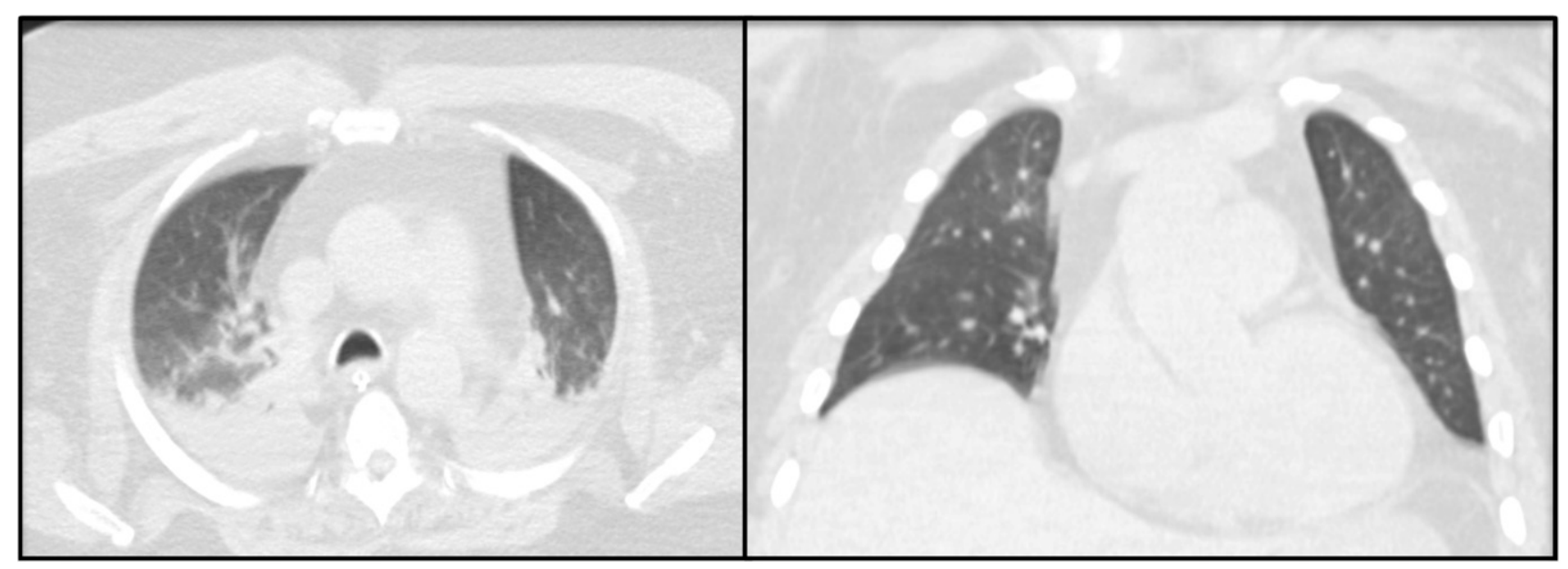

FIG. 1. Admission CT showing no evidence of pulmonary herniation.

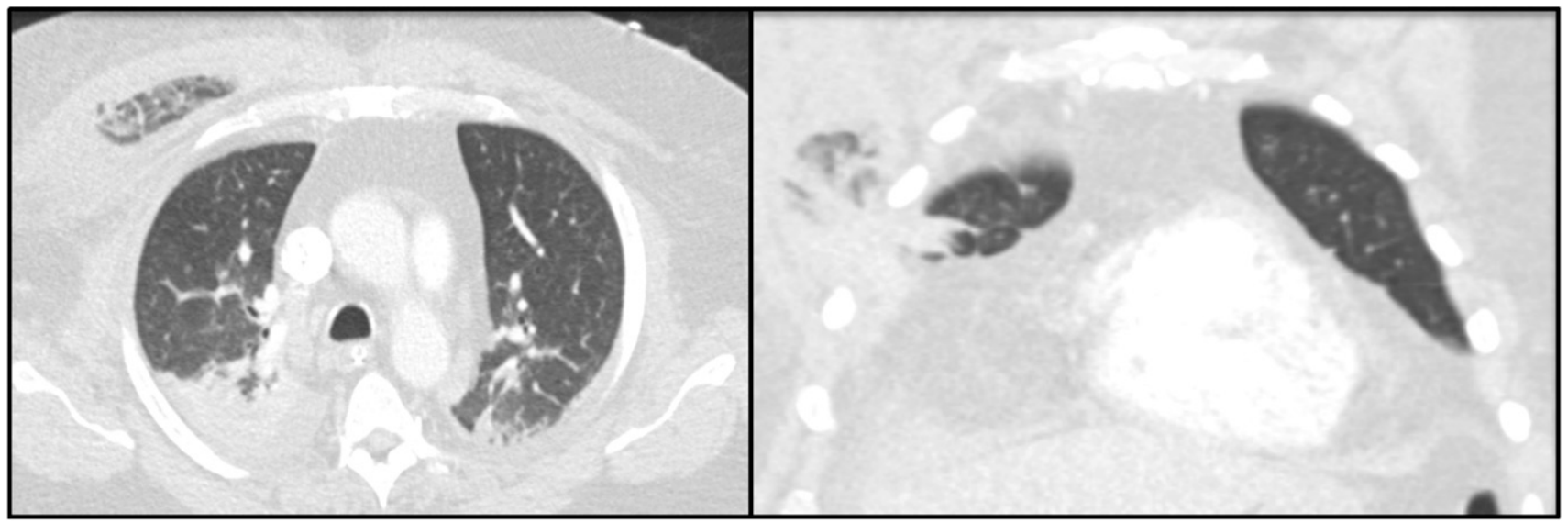

FIG. 2. CT obtained hospital day 4 with evidence of herniation of the patient's right middle lobe through widened anterior fourth intercostal space.

not result in the resolution of the underlying pulmonary contusion. This case clearly illustrates that lung herniation can be a lethal sequelae of blunt thoracic trauma despite appropriate surgical reduction of the hernia.

Rebecca Brown, M.D. Anthony G. Charles, M.D., M.P.H.

\section{University of North Carolina} Chapel Hill, North Carolina

\section{REFERENCES}

1. Montgomery JG, Lutz H. Hernias of the lung. Ann Surg 1925;82:220-31.

2. Clark AJ, Hughes N, Chisti F. Traumatic extrathoracic lung herniation. Br J Radiol 2009;82L:e82-4.

3. Detorakis EE, Androulidakis E. Intercostal lung herniationthe role of imaging. J Radiol Case Rep 2014;8:16-24.

4. François B, Desachy A, Cornu E, et al. Traumatic pulmonary hernia: surgical versus conservative management. J Trauma 1998; 44:217-9. 